\title{
PRIORITY COMMUNICATION
}

\section{Influence of the Generation of Mesopores on the Hydroisomerization Activity and Selectivity of $n$-Hexane over Pt/Mordenite}

\author{
M. Tromp, J. A . van B okhoven, M. T. G arriga O ostenbrink, J. H . Bitter, \\ K. P. de Jong, and D. C. Koningsberger ${ }^{1}$ \\ D epartment of Inorganic Chemistry and Catalysis, D ebye Institute, U niversity of U trecht, PO B ox 80083, 3508 TB U trecht, The N etherlands \\ E-mail: D.C.Koningsberger@chem.uu.nl
}

R eceived D ecember 1, 1999; accepted D ecember 9, 1999

\begin{abstract}
The activity and selectivity of hydroisomerization of $n$-hexane over Pt/mordenite is strongly influenced by acid leaching. Theactivity increases and the selectivity changes to favor primary products. Leaching selectively modifies the mordenite structure, making more sites accessible for reaction and facilitating desorption of reaction products. It is argued that the activity of untreated mordenite is limited by mass transfer effects. These effects largely vanish after modification of the zeolite structure by generation of a 3-D micropore structure as well as mesopores. The alleviation of intracrystalline diffusion limitation is themajor factor in activity enhancement after acid leaching of $\mathrm{Pt} /$ mordenite.

(c) 2000 A cademic Press
\end{abstract}

\section{INTRODUCTION}

Modern combustion engines require a fuel with a high research octane number ( $\mathrm{RON})$. The naphtha directly obtained by distillation of crude oil cannot meet this requirement. A n ecologically sound way to increase the RON of a fuel is by increasing the concentration of branched alkanes at the expense of linear alkanes. To achieve this one of the major industrial processes, besides the alkylation of alkanes, is the (hydro)isomerization of linear alkanes to branched ones.

$H$ ydroisomerization requires a bifunctional catalyst, consisting of a (de)hydrogenating metal function and a protonating acid function (1). In the first reaction step, the alkane is dehydrogenated to an alkene on the noble metal. A fter migration of the alkenes to the acid site, protonation takes place and the carbocation reacts to a branched carbocation (2) via a cyclopropyl intermediate. This is usually the ratedetermining step of the reaction. The formed carbocation is deprotonated and after migration to a metal site, it is hydrogenated yielding a branched alkane. B efore deprotonation, the carbocation can undergo another isomerization

\footnotetext{
${ }^{1}$ To whom correspondence should be addressed.
}

step forming a dibranched product (with a higher RON) or unwanted cracking products. The formed isoalkanes can readsorb onto an acid site, initiating a new isomerization cycle or forming cracking products.

Two commercial catalytic processes exist for hydroisomerization (3). O ne is based on the use of a chlorinated $\mathrm{Pt} / \mathrm{Al}_{2} \mathrm{O}_{3}$ catalyst while the other one is based on Pt/ mordenite. The Pt/mordenite system is more stable (less sensitive to sulfur and water) but it is less active than the chlorinated $\mathrm{Pt} / \mathrm{A} \mathrm{I}_{2} \mathrm{O}_{3}$. Therefore, higher temperatures $(523 \mathrm{~K})$ are needed, which is thermodynamically unfavorable for the formation of (di)branched products.

A s mentioned above, $\mathrm{Pt} /$ mordenite is very suitable for the hydroisomerization reaction. I t is well known that leaching and steaming of mordenite significantly alter the activity of $\mathrm{Pt} /$ mordenite $(4,5)$. A fter dealumination by acid leaching treatments an optimum Si/A I ratio was found for the hydroisomerization of pentane $(4,6)$. A scientific understanding of these findings is still lacking. The optimum Si/A I ratio is very important for industrial applications since products with a high R ON have to be obtained, which demands optimal catalytic performance.

This study is part of an extended investigation of the influence of acid leaching and steaming treatments on the catalytic activity and selectivity of Pt/mordenite. Here, we focus on the increase in activity after acid leaching. This is studied by the determination of the catalytic properties and different characterization measurements $\left(\mathrm{N}_{2}\right.$ physisorption, determination of acid site density, TE M ) of untreated crystalline mordenite and acid-leached mordenite. It will be shown that acid leaching generates mesopores, which make acid sites more accessible for reaction and facilitate desorption of reaction products from the micropore surface. In other words, acid leaching decreases diffusion limitation effects.

Extraframework $\mathrm{Al}$ is produced predominantly after steaming. Its influence on the activity and selectivity is discussed in a forthcoming paper. 


\section{METHODS}

Twenty-five grams of $\mathrm{Na}$ /mordenite (obtained from A moco, LZM -5) with 4.6 wt\% $\mathrm{Na}$ and an aluminum content of 5.7 wt\% was exchanged three times with $300 \mathrm{ml}$ of $1 \mathrm{M} \mathrm{NH}_{4} \mathrm{NO}_{3}$ solution for $24 \mathrm{~h}$ at $353 \mathrm{~K}$. The formed $\mathrm{NH}_{4}$ /mordenite was washed, filtered, and dried overnight at $393 \mathrm{~K}$. The dried mordenite was calcined, removing $\mathrm{NH}_{3}$ and creating $\mathrm{H} /$ mordenite, by heating to $773 \mathrm{~K}$ at a rate of $1 \mathrm{~K} \mathrm{~min}^{-1}$ in a $50 \mathrm{ml} \mathrm{min} \mathrm{m}^{-1} \mathrm{~N}_{2} / \mathrm{O}_{2}$ (80/20) flow and was maintained at that temperature for $3 \mathrm{~h}$ and subsequently cooled down to room temperature. This mordenite is the parent mordenite (MOR 5.7).

L eaching of the parent mordenite with $1 \mathrm{M} \mathrm{HCl}$ for one hour gave an $\mathrm{H}$ /mordenite with a lower aluminum amount of 2.6 wt\% A I (MOR 2.6).

Platinum loading (2 wt\%) of the mordenites was performed by ion exchange at room temperature for $24 \mathrm{~h}$ with a highly diluted platinum tetraammonium hydroxide (A Idrich) solution. The exchanged mordenite was washed, filtered, and dried overnight at $393 \mathrm{~K}$. The dried sample was calcined in a $50 \mathrm{ml} \mathrm{min}^{-1} \mathrm{~N}_{2} / \mathrm{O}_{2}$ flow at $723 \mathrm{~K}$ for $2 \mathrm{~h}$ at a rate of $0.5 \mathrm{~K} \mathrm{~min}^{-1}$ and cooled to room temperature.

$\mathrm{N}$ itrogen adsorption and desorption isotherms were measured using a M icromeritics A SA P 2400. Temperature programmed desorption (TPD) and simultaneous thermogravimetric analysis (TGA) experiments were carried out with $n$ - and isopropylamine. The TPD/TGA measurements were performed using a Perkin-E Imer T G S-2 microbalance connected to a Fison Instruments quadrupole mass spectrometer.

$\mathrm{H}$ igh resolution transmission electron microscopy (HRTEM) was performed using a Philips CM 30 T electron microscope, with an $\mathrm{LaB}_{6}$ filament as the source of electrons, operating at $300 \mathrm{kV}$. Samples were mounted on a microgrid carbon polymer supported on a copper grid by placing a few droplets of a suspension of ground sample in ethanol on the grid, followed by drying at ambient conditions.

$\mathrm{H}$ ydroisomerization of $\mathrm{n}$-hexane was carried out in a glass tube reactor placed in a thermostatic tube oven. The pressure of the system was controlled by a back-pressure regulator at 1 bar. M ass flow controllers regulated the in- coming gas flows. The $n$-hexane (M erck) was injected into the system using a high performance liquid chromatograph pump (Shimadzu L C-10A D). The reaction products were analyzed using a Perkin-E Imer A utoSystem X L gas chromatograph with a Chrompack capillary column (CP Sil 5 CB $0.53 \mathrm{~mm} 50 \mathrm{~m}$ ). The whole system was heated to avoid condensation of gases.

Prior to catalytic testing the samples were reduced in situ at $623 \mathrm{~K}$ for $1 \mathrm{~h}$ (at a rate of $5 \mathrm{~K} \mathrm{~min}^{-1}$ and subsequently cooled to operating temperature in $\mathrm{H}_{2}$. Conversion plots were measured using $\sim 100 \mathrm{mg}$ of catalyst, with particle size of 150-500 $\mu \mathrm{m}, \mathrm{n}$-hexane flow of $0.118 \mathrm{mmol} \mathrm{min}^{-1}$, and $\mathrm{H}_{2}$ flow of $25 \mathrm{ml} \mathrm{min} \mathrm{mol}^{-1}\left(\mathrm{H}_{2}: \mathrm{C}_{6}\right.$ ratio $\left.9 \mathrm{~mol} / \mathrm{mol}\right)$ at a total pressure of 1 bar. The temperature in these experiments was increased from $423 \mathrm{~K}$ at a rate of $0.5 \mathrm{~K} \mathrm{~min}^{-1}$ to $623 \mathrm{~K}$. Contact time variations were carried out at $523 \mathrm{~K}$ and $\mathrm{a}$ pressure of 1 bar. To be able to measure all samples at low conversions $(<5 \%)$, different amounts of catalyst and different $\mathrm{n}$-hexane and $\mathrm{H}_{2}$ flows were used (constant $\mathrm{H}_{2}: \mathrm{C}_{6}$ ratio $9 \mathrm{~mol} / \mathrm{mol}$ ). With the equipment used it was possible to measure conversions from 0.2 to $100 \%$ with about $0.05 \%$ accuracy.

\section{RESULTS}

\section{$\mathrm{N}_{2}$ Physisorption M easurements}

The physicochemical parameters of the samples are compiled in Table 1. T-plot analysis of the $\mathrm{N}_{2}$ sorption isotherms shows that after leaching the mesoporous surface area increased and a mesopore volume formed.

\section{D etermination of A cid Site D ensity}

The number of acid sites determined with TPD/TGA of $\mathrm{n}$ - and isopropylamine decreased after acid leaching. A discrepancy between the values obtained with $\mathrm{n}$ - and isopropylamine was found for M O R 5.7. For this sample the number of acid sites probed by isopropylamine was only $\sim 2 / 3$ of the amount measured by $n$-propylamine.

\section{TEM}

In Fig. 1 HRTEM pictures of MOR 5.7 and MOR 2.6 are given. The primary crystal size of MOR 5.7 is about

\section{TABLE 1}

\section{Physicochemical Properties of the Mordenites}

\begin{tabular}{lccccc}
\hline Sample & $\begin{array}{c}\text { Mesoporous surface } \\
\text { area }\left(\mathrm{m}^{2} / \mathrm{g}\right)\end{array}$ & $\begin{array}{c}\text { M icropore } \\
\text { volume }(\mathrm{cc} / \mathrm{g})\end{array}$ & $\begin{array}{c}\text { Mesopore } \\
\text { volume }(\mathrm{cc} / \mathrm{g})\end{array}$ & $\begin{array}{c}\text { A cid sites by n-propylamine } \\
\text { decom. }(\mathrm{mmol} / \mathrm{g})\end{array}$ & $\begin{array}{c}\text { A cid sites by isopropylamine } \\
\text { decom. }(\mathrm{mmol} / \mathrm{g})\end{array}$ \\
\hline M OR 5.7 & 30 & 0.19 & 0.04 & 2.1 & 1.4 \\
M OR 2.6 & 89 & 0.18 & 0.11 & 1.1 & 1.1 \\
\hline
\end{tabular}

\footnotetext{
$2.1 \mathrm{mmol} \mathrm{g}^{-1} \mathrm{Al}$.

b $1.0 \mathrm{mmol} \mathrm{g}^{-1} \mathrm{Al}$.
} 
FIG. 1. (A) HRTEM picture of MOR 5.7; (B) HRTEM picture of MOR 2.6.

$0.4 \times 0.2 \mu \mathrm{m}$. The primary crystallites agglomerated to larger clusters, with the crystallites orderly packed parallel to each other. A fter leaching, light spots and lines appear all over the primary crystallites, indicating the disappearance of zeolite material. The edges of the crystals are rounded, but the primary crystals can still be recognized.

\section{Catalytic Testing}

Variation of the Pt loading revealed that at 2 wt $\%$ the reaction is not limited by Pt. Conversion measurements performed with different flows and amounts of catalyst, at constant contact times, confirmed that no external diffusion limitations took place. Finally, measuring conversion at a constant temperature of $523 \mathrm{~K}$ and a constant n-hexane flow of $0.118 \mathrm{mmol} \mathrm{min}^{-1}$ and $\mathrm{H}_{2}$ flow of $25 \mathrm{ml} \mathrm{min}^{-1}$ at low conversions ( $5 \%$ ) indicated that no deactivation took place.

By plotting the yield of products against the conversion of $n$-hexane (determined by contact time measurements ( 0.2 to about $5 \%$ conversion) at $523 \mathrm{~K}$ ), the primary and secondary products were determined. The 2- and the 3methylpentanes were analyzed as primary products, the dibranched butanes as secondary products, and cracking products as both primary and secondary.

In Table 2 the relative activities of the Pt/mordenites at $1 \%$ conversion of $\mathrm{n}$-hexane at $523 \mathrm{~K}$ and 1 bar are given. The relative activity of the catalyst was determined from the contact time at $1 \%$ conversion of $n$-hexane. The (relative) activity per gram increased after acid leaching,

\section{TABLE 2}

Relative Activity and Selectivity of the D ifferent Products for $n$-hexane I somerization of $\mathrm{Pt} / \mathrm{M}$ ordenites at $523 \mathrm{~K}, 1 \%$ C onversion, $p=1$ bar

\begin{tabular}{ccccc}
\hline Sample & $\begin{array}{c}\text { Relative } \\
\text { activity }\end{array}$ & $\begin{array}{c}\text { Selectivity to } \\
\text { monobranched }\end{array}$ & $\begin{array}{c}\text { Selectivity to } \\
\text { dibranched }^{\mathrm{a}}\end{array}$ & $\begin{array}{c}\text { Selectivity } \\
\text { of cracking }^{\text {ch }}\end{array}$ \\
\hline M OR 5.7 & 1.0 & 70 & 11 & 19 \\
M OR 2.6 & 7.5 & 82 & 7 & 13 \\
\hline
\end{tabular}

a Selectivity in $\% \mathrm{~mol}$. 


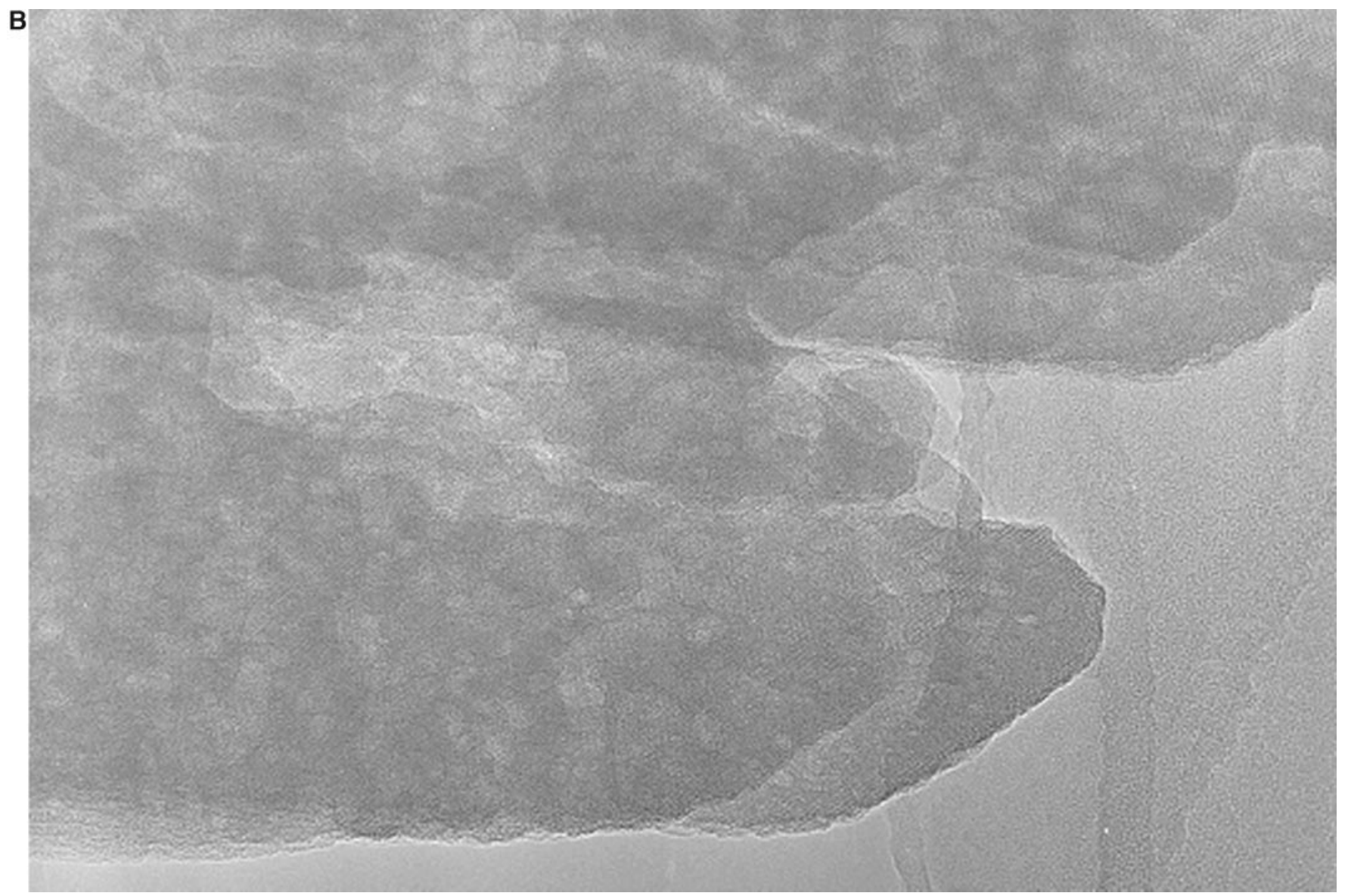

FIG. 1-Continued

corresponding to the first increase in the activity given in the literature, in which an optimum was found for the activity as a function of the dealumination treatments $(4,6)$.

B oth samples showed a high selectivity to the monobranched isomers, but dibranched butanes and cracking products were also formed. The selectivity toward monobranched products increased after acid leaching and the selectivity to dibranched and cracking products decreased.

\section{E stimation of Thiele M odulus}

The Thiele modulus of the parent mordenite, M OR 5.7, was calculated using the crystal size determined from HRTEM $(0.2 \times 0.4 \mathrm{~m})$ at $1 \%$ conversion at $523 \mathrm{~K}$. First, the volumetric first order rate coefficient $\left(\mathrm{k}_{\mathrm{v}}\right)$ was calculated using the equation

$$
k_{\vee} \equiv-\frac{\phi_{\vee}}{V_{\mathrm{Cr}}} \ln (1-x)
$$

where $\phi_{\mathrm{V}}$ is the total volumetric flow rate comprising both $\mathrm{n}$-hexane $\left(3.3 \times 10^{-7} \mathrm{~m}^{3} \mathrm{~s}^{-1}\right)$ and $\mathrm{H}_{2}\left(3.2 \times 10^{-6} \mathrm{~m}^{3} \mathrm{~s}^{-1}\right), \mathrm{V}_{\mathrm{cr}}$ is the total volume of the catalyst crystals $\left(1.4 \times 10^{-8} \mathrm{~m}^{3}\right)$, and $\mathrm{x}$ is the measured conversion (0.01). A volumetric first order rate coefficient of $2.5 \mathrm{~s}^{-1}$ was found. Subsequently, the Thiele modulus was calculated using the equation

$$
\phi \equiv L \sqrt{\frac{k_{\vee}}{D_{\mathrm{e}}}},
$$

where $L$ is the effective diffusion length $(0.2 \mathrm{~m})$ and $D_{e}$ is the diffusion coefficient. It is known that the 12-membered ring channels in mordenite are oriented along the length of the crystal (7).

A Thiele modulus of 3.2 was found assuming an effective diffusion coefficient of $10^{-14} \mathrm{~m}^{2} \mathrm{~s}^{-1}$, leading to an effectiveness factor of 0.3 .

\section{DISCUSSION}

$\mathrm{N}_{2}$ Physisorption M easurements and TE M

The BET analysis of the $\mathrm{N}_{2}$ physisorption studies on the different mordenites showed a clear increase in both the mesopore surface and volume with acid leaching. This indicates a partial destruction of the zeolite structure during acid leaching. These results are in agreement with the HRTEM pictures of the leached mordenite that showed 
light spots and lines all over the crystal, indicating the disappearance of zeolite material. These spots are interpreted as mesopores and/or cracks formed in the crystals after acid leaching while the crystallite shape is maintained. The destruction of the mordenite structure, together with the formation of mesopores by acid leaching, has already been mentioned in the literature $(5,8,9)$.

\section{A cid Site D ensity}

The decomposition reactions of both $n$ - and isopropylamine were found to be excellent tools for determining the number of B rønsted acid sites in zeolites in order to distinguish different pore sizes (10). The assumption is made that onen-or isopropylamine molecule probes one acid site. The number of acid sites of $2.1 \mathrm{mmol} \mathrm{g}{ }^{-1}$ catalyst determined for M OR 5.7 by n-propylamine corresponded well with the total aluminum content in M OR 5.7 of $2.1 \mathrm{mmol} \mathrm{g}$. This indicates that all aluminum was involved in the formation of B rønsted acid sites. N ote that for M O R 5.7 a discrepancy was observed in the numbers of B rønsted acid sites determined by $n$ - and isopropylamine. The number determined with isopropylamine is $\sim 2 / 3$ of the number determined with n-propylamine.

M ordenite consists of straight 12-membered ring channels in the $c$ direction with a slightly elliptical cross section of $6.7 \times 7.0 \AA^{\circ}$. The main channels possess $4.8-A^{\circ}$-deep side pockets in the $b$ direction with an aperture of $2.6 \times 5.7 A^{\circ}$ (8-membered ring). In the literature it has already been mentioned that $\sim 2 / 3$ of the B rønsted acid sites are located in the 12-membered ring (11). It is also known that isopropylamine does not completely fit into the 8-membered ring of a FER, in contrast to n-propylamine (10). Therefore, it is logical to assume that in M OR 5.7, the acid sites in the 8-membered rings are undetected by isopropylamine. The number of acid sites probed by isopropylamine corresponds well with the fact that $\sim 2 / 3$ of the total number of acid sites are located in the 12-membered rings.

A fter acid leaching both probe molecules gave an identical acid site density, so the acid sites located in the 8membered rings, i.e., in the side pockets, have been made accessible for larger molecules such as isopropylamine or could have been selectively removed. The decrease of the numbers of acid sites after leaching is consistent with the decrease of the aluminum content in the mordenite. The number of acid sites determined with propylamine is $1.1 \mathrm{mmol}$ $\mathrm{g}^{-1}$, which is within $10 \%$ of agreement with the amount of aluminum in MOR 2.6, $1.0 \mathrm{mmol} \mathrm{g}^{-1}$.

\section{Catalytic Performance}

The structural modifications in the mordenite are accompanied by changes in its catalytic properties, both in activity and in selectivity. The selectivity shifts from dimethylbutanes and cracking products to monomethylpentanes after acid leaching. So after leaching, the selectivity for primary products increases while the secondary products are suppressed. The decrease in secondary reactions can be explained by faster desorption of products from the micropore surface.

The different characterization methods showed that acid leaching partially destroyed the structure. The acid leaching treatment selectively opens the side pockets so that all the B rønsted acid sites become accessible for isopropylamine. $B$ ased on kinetic diameters we assume that if an isopropylamine can probe an acid site, enough space is available for an n-hexane to react on this acid site via a bulky cyclopropyl intermediate to a methylpentane and desorb from the surface. So after leaching, all B rønsted acid sites present in the mordenite participate in the reaction. A shas been proposed by OIken and $G$ arces and by M eima, a 3-D pore structure is formed in the mordenite $(8,9)$. A local destruction of the mordenite structure by growing metal particles to form a 3-D mordenite, making more active sites accessible for reaction (12), has also been proposed.

A Ithough the number of acid sites probed with isopropylamine, i.e., the number of accessible sites for MOR 2.6, is less than that for M OR 5.7, the activity per gram of catalyst for M O R 2.6 is higher. It seems that not all iso propylamineaccessible sites in the 12-membered ring in MOR 5.7 participate equally in the hydroisomerization reaction. The estimated Thiele modulus for M OR 5.7 showed the possibility of diffusion limitations of $n$-hexane in the mordenite. The value for the diffusion coefficient of $10^{-14} \mathrm{~m}^{2} \mathrm{~s}^{-1}$ for n-hexane was assumed to be reasonable for intracrystalline diffusion in mordenite pores as reported (13). Moreover, the calculation was made for the diffusion of $n$-hexane, while other reaction products in the pores are likely to influence diffusion. The bulkier (branched) products diffuse even slower than $n$-hexane, since their kinetic diameter $\left(\sim 5 A^{\circ}\right)$ (14) and the diameter of the mordenite pores $\left(\sim 7 A^{\circ}\right)$ make it difficult for these products to pass any of the other molecules. It is clear that the creation of mesopores due to acid leaching results in better accessibility of the B rønsted acid sites and easier desorption of products from the surface due to increased external surface, explaining the major part of the increase in both activity and selectivity.

\section{CONCLUSIONS}

B oth the activity and the selectivity for the hydroisomerization reaction for $\mathrm{Pt}$ /mordenite change after acid leaching of the mordenite. Diffusion limitation in untreated crystalline MOR 5.7 is established. A cid leaching makes the catalyst more active, while increasing external surface and creating more mesopores and/or cracks. M ore acid sites become accessible for reaction and desorption from the surface facilitates, which decreases diffusion limitations. D ue to smaller diffusion limitations and easier desorption from the surface after leaching, the residence time of products 
on the surface decreases so that fewer secondary reaction products are formed. This results in an increase of the selectivity to monobranched products.

\section{ACK NOWLEDG MENTS}

We thank J. A . Z. Pieterse, U niversity of Twente, D epartment of C ataIytic Processes and Materials, Enschede, The Netherlands, for helpful discussions; D r. P. J. Kooyman, $\mathrm{N}$ ational Centre for $\mathrm{H}$ igh R esolution E lectron M icroscopy, D elft U niversity of Technology, The Netherlands, for performing the electron microscopy investigations; and D r. J. T. Miller, B P A moco R esearch Center, Naperville, IL, for providing the samples. This work was supported by the Catalysis Center $U$ trecht $(N L)$ and by the Technology Foundation and was carried out in connection with the $\mathrm{N}$ etherlands Institute for Catalysis R esearch N IOK.

\section{REFERENCES}

1. Weisz, P. B., A dv. Catal. 13, 137 (1962).

2. Weitkamp, J., in "Proceedings, 7th I nternational C onference on CataIysis, Tokyo, 1980" (T. Seiyama and K. Tanabe, E ds.), p. 1404. E Isevier, A msterdam, 1981.
3. Heinemann, H., in "Catalysis, Science and Technology" (J. R. A nderson and M. Boudart, E ds.), Vol. 1, p. 1. Springer-Verlag, B erlin, 1981.

4. Corma, A ., and M artinez, A ., in "C atalytic A ctivation and F unctionalisation of L ight A Ikanes: A dvances and Challenges" (E . G. D erouane, J. H aber, F. Lemos, F. R . R ibeiro, and M . G uisnet, E ds.), p. 35. K luwer A cademic, D ordrecht, 1998.

5. Stach, H., Jänchen, J., Jerschkewitz, H. G., Lohse, U., Parlitz, B., Zibrowius, B., and H unger, M., J. Phys. Chem. 96, 8473 (1992).

6. Koradia, P. B., Kiovsky, J. R., and A sim, M. Y., J. Catal. 66, 290 (1980).

7. M eier, W. M ., Z. K ristallogr. 115, 439 (1961).

8. Olken, M. M., and Garces, J. M., in "Proceedings from the 9th International Z eolite Conference" ( $R$. von B allmoos, J. B. H iggins, and M. M. J. Treacy, E ds.), p. 559. B utterworth-H eineman, B oston, 1992.

9. M eima, G. R., CATTECH 2(1), 5 (1998).

10. Palkhiwala, A. G., and G orte, R. J., Catal. L ett. 57, 19 (1999).

11. A Iberti, A ., Z eolites 19, 411 (1997).

12. Carvil, B. T., Lerner, B. A., A delman, B. J., Tomczak, D. C., and Sachtler, W. M. H., J. Catal. 144, 1 (1993).

13. Post, M . F. M ., Stud. Surf. Sci. Catal. 58, 391 (1991).

14. B reck, D. W., "Zeolite Molecular Sieves: Structure, Chemistry and U se," Wiley, N ew York, 1974. 